\title{
Multimodal treatment options for bilobar colorectal liver metastases
}

\author{
Kia Homayounfar • Torsten Liersch • Martin Niessner • \\ Johannes Meller • Thomas Lorf • Heinz Becker • \\ B. Michael Ghadimi
}

Received: 3 December 2009 /Accepted: 27 January 2010/Published online: 7 March 2010

(C) The Author(s) 2010. This article is published with open access at Springerlink.com

\begin{abstract}
Purpose We evaluated individualized multimodal oncological strategies in patients with bilobular colorectal liver metastases (biCRC-LM) as well as their effect on R0 resection rates, disease-free survival (DFS), and overall survival (OS). Methods Between January 2001 and December 2008, 64 patients were assigned to straightforward or two-stage liver resection \pm preoperative 5-fluorouracil (5FU)-based chemotherapy (CTx). Postoperative strategy after R0-resection was either "wait and see" or "adjuvant" therapy (3 cycles of CTx or anti-carcinoembryonic antigen (CEA)-radioimmunotherapy with ${ }^{131}$ I-labetuzumab in a dose of $40-50 \mathrm{mCi} / \mathrm{m}^{2}$ ).

Results Forty-three initially unresectable patients received preoperative CTx for downsizing of their biCRC-LM. Straightforward or two-stage liver resection was intended in 40 and 24 patients, respectively. Histopathologically confirmed R0-liver resection could be achieved in 47 patients. Surgical morbidity and mortality rates were $33 \%$ and $1.5 \%$, respectively. Postoperatively, 26 patients received anti-cancer therapy $(5 \times \mathrm{CTx}, 21 \times$ anti-CEA-radioimmunotherapy). After R0-liver resection, median OS was significantly better
\end{abstract}

K. Homayounfar and T. Liersch contributed equally to this manuscript and study.

K. Homayounfar $(\bowtie) \cdot$ T. Liersch $\cdot$ M. Niessner $\cdot$ T. Lorf $\cdot$

H. Becker $\cdot$ B. M. Ghadimi

Department of General and Visceral Surgery,

University Medical Centre, Georg-August University Goettingen,

Robert-Koch-Strasse 40,

37073 Goettingen, Germany

e-mail: khomayounfar@chirurgie-goettingen.de

J. Meller

Department of Nuclear Medicine, University Medical Centre,

Georg-August University Goettingen,

Robert-Koch-Strasse 40,

37073 Goettingen, Germany compared to R1/R2 resections followed by palliative 5FUCTx (38 versus 19 months, $p=0.035$ ). There was no significant difference in DFS $(p=0.650)$ and OS $(p=0.435)$ between straightforward and two-stage liver resection. Compared to "wait and see" strategy, the application of postoperative therapy in adjuvant intent was associated with a better OS $(p=0.048)$.

Conclusion Extensive liver resection within multimodal treatment concepts is justified in patients with biCRC-LM when complete resection of all metastases seems to be achievable.

Keywords Multimodal treatment . Two-stage hepatectomy · Portal vein ligation · Oncological strategy . Adjuvant therapy

\section{Introduction}

Only histopathologically confirmed R0 liver resection (LR) of colorectal liver metastases (CRC-LM) offers a long-term survival, and 5-year-survival rates up to $58 \%$ have been reported from specialized centers [1, 2]. Patients with bilobar colorectal liver metastases (biCRC-LM) represent a particular challenge as their prognosis is extremely poor. If complete (R0) LR can be achieved in cases of primary resectability, even these patients benefit from an extended surgical intervention and experience a 5-year-survival rate up to $20 \%$ [3]. Unfortunately, up to $70 \%$ of patients with biCRC-LM are primarily unresectable even when assessed by an experienced hepatobiliary surgeon. These patients represent an advanced stage of disease with a 5-year-survival rate below $5 \%$ under palliative polychemotherapy $[4,5]$. In the last decade, individualized multimodal approaches using preoperative chemotherapy and/or two-stage procedures 
including portal vein occlusion (PVO) have been established aiming to enhance the proportion of initially unresectable patients with biCRC-LM suitable for secondary R0-LR.

The potential of preoperative chemotherapy has recently been demonstrated by the randomized multicenter CELIMstudy [6], which confirmed a $28 \%$ secondary R0-resection rate following preoperative 5 -fluorouracil (5FU)-based chemotherapy extended by cetuximab and either oxaliplatin or irinotecan. Furthermore, the advanced surgical approach of two-stage hepatectomy with PVO also demonstrated encouraging secondary resection rates of $58-75 \%[7,8]$. However, as unresectability might be caused not only by bilobar metastatic spread but also by unilobar disease with small left lateral liver segments or close relation of CRC-LM to major intrahepatic structures, the rather technical stratification of primary unresectability neglects the different oncological stage of unilobar and bilobar CRC-LM. Recently, Nikfarjam and colleagues reported a series of 64 patients with CRCLM treated within a multimodal treatment concept and confirmed bilobar spread $(n=40)$ to be an independent prognostic factor associated with poorer disease-specific survival $(p=0.02)$ [9]. Besides these results, studies on multimodal treatment strategies and their oncological outcome in patients with biCRC-LM are still lacking.

Therefore, the aim of the present study was to analyze our single-center experience with multimodal strategies including innovative-targeted therapies like anti-carcinoembryonic antigen (CEA)-radioimmunotherapy $[10,11]$ on resection rates and survival in patients with biCRC-LM.

\section{Patients and methods}

Study population

Out of 168 consecutive patients surgically treated for CRC-LM between January 2001 and December 2008, 64 presented with bilobar hepatic involvement diagnosed by pretherapeutical staging. These patients reflect the study population of the present investigation. Oncological treatment, perioperative data, and survival were retrospectively analyzed based on a prospectively designed database.

The treatment rationale for patients with biCRC-LM was first to evaluate whether all liver metastases were resectable by straightforward resection. If that was not possible, we assessed the possibility of complete resection by a twostage procedure with portal vein ligation. Those patients that seemed to be unresectable even with a two-stage concept were treated by preoperative systemic 5FU-based chemotherapy and were re-evaluated for secondary resectability (straightforward or two-stage resection) every three courses.
Clinicopathologic data concerning surgery and adjuvant therapy of the primary tumor are summarized in Table 1. Of the patients, $47(74 \%)$ had locally advanced and distant metastatic disease stages $(8 \times$ UICC III, $39 \times$ UICC IV $)$ at the time of primary tumor resection. CRC-LM were classified as synchronous when they had been diagnosed prior to, during, or up to 3 months after surgery for the primary tumor. The mean disease-free survival (DFS) between primary tumor resection and diagnosis of first CRC-LM was $7 \pm 2.9$ (range 0-69) months for all patients. One patient presented with intrahepatic recurrence.

Staging procedures before liver resection

Pretherapeutical staging included clinical examination, chest $\mathrm{X}$-ray, abdominal computed tomography (CT) or magnetic resonance imaging, and serum level of CEA. As we observed extrahepatic metastases early after LR which presumably were existent but undetected at the time of LR, staging procedures were extended to a thoracoabdominal computed tomography as well as a ${ }^{18} \mathrm{~F}$-fluorodeoxyglucose-positron emission tomography in January 2006. All staging data were reviewed by a board of experienced surgical and medical oncologists and hepatobiliary surgeons.

Table 1 Clinicopathologic data of primary tumor therapy

\begin{tabular}{|c|c|c|}
\hline \multirow[t]{2}{*}{ Parameter } & \multicolumn{2}{|c|}{ Patients } \\
\hline & No. & $\%$ \\
\hline \multicolumn{3}{|l|}{ Sex } \\
\hline Female & 18 & 28 \\
\hline Male & 46 & 72 \\
\hline \multicolumn{3}{|l|}{ Primary cancer } \\
\hline Colon & 39 & 61 \\
\hline Rectum $^{\mathrm{a}}$ & 25 & 39 \\
\hline \multicolumn{3}{|c|}{ Primary tumor stage at surgery } \\
\hline UICC I & 4 & 6 \\
\hline UICC II & 13 & 20 \\
\hline UICC III & 8 & 13 \\
\hline UICC IV & 39 & 61 \\
\hline \multicolumn{3}{|c|}{ Adjuvant 5FU-based chemotherapy } \\
\hline Yes & 15 & 23 \\
\hline No & 49 & 77 \\
\hline \multicolumn{3}{|c|}{ Detection of hepatic metastases } \\
\hline Synchronous ${ }^{\mathrm{b}}$ & 42 & 66 \\
\hline Metachronous & 22 & 34 \\
\hline
\end{tabular}

UICC Union Internationale Contre le Cancer

${ }^{\mathrm{a}} \mathrm{Up}$ to $16 \mathrm{~cm}$ above anocutaneous verge as measured by rigid rectoscopy according to UICC/TNM 2003

${ }^{\mathrm{b}} \leq 3$ months between primary tumor resection and diagnosis of liver metastases 
Anti-tumor therapy

Preoperative systemic 5FU-based chemotherapy was applied for both downsizing of primary unresectable CRC-LM and assessment of tumor responsiveness. Response to preoperative chemotherapy was measured by an experienced radiologist using the Response Evaluation Criteria in Solid Tumors (RECIST) [12]. In accordance with these criteria, partial remission had been defined as at least 30\% decrease and progressive disease as at least $20 \%$ increase in the sum of the longest diameter of target lesions. Stable disease was present in any change of size between these two values.

LR was performed according to established surgical standard operating procedures. In those patients needing LR of more than four segments due to the distribution of their CRC-LM, the future remnant liver volume was calculated preoperatively according to Truant et al. [13] based on CT scan volumetric estimation. In cases with a future remnant liver weight to body weight ratio less than $0.5 \%$ two-stage hepatectomy with portal vein ligation (PVL) as previously described [7] was indicated to preserve sufficient liver function. During the phase of liver hypertrophy, no chemotherapy was administered. The use of radiofrequency ablation (RFA) was limited to two different settings: first, when resection of a single or multiple metastases was technically impossible. In these cases, operation was classified as incomplete (R1) regardless of simultaneous complete (R0) resection of other CRC-LM. Second, when LR was canceled due to intrahepatic tumor progression, but RFA was assumed to be beneficial for local tumor control. Those patients that experienced surgical exploration \pm PVL without subsequent LR were classified as incomplete (R2) resection. Postoperative liver insufficiency was defined by prothrombin time $<50 \%$ and serum bilirubin $>50 \mu \mathrm{mol} / \mathrm{l}$ on post-op day 5 as described by Balzan et al. [14].

During the study term, the Association of Scientific Medical Societies in Germany interdisciplinary guidelines for the treatment of CRC-LM [15] recommended a "wait and see" strategy after R0-LR, which had been the standard for our study population. However, over the same period of time, we performed a phase II trial investigating the feasibility and safety of single or repeated anti-CEA-radioimmunotherapy with ${ }^{131}$ I-labetuzumab (hMN-14 humanized anti-CEA immunoglobulin G1 - subclass monoclonal antibody, Immunomedics Inc. Morris Planes NJ, USA) after R0-resection of CRC-LM on DFS and overall survival (OS) [10, 11]. Therefore, eligible patients with immunohistochemically proven CEA-overexpression on CRC-LM cells were enrolled even after preoperative 5FU-based chemotherapy while the study was open for recruitment. Alternatively, patients requesting adjuvant therapy were treated by systemic 5FUbased chemotherapy according to the investigators' discretion (FOLFOX or FOLFIRI regimen).
Statistics

Median follow-up was 23 (range 1-83) months. Statistical comparisons between distinct groups were performed by Fisher's exact test. The baseline for survival analyses was the time point of liver resection with removal of the $\mathrm{CRC}$ LM. In patients subjected to two-stage procedures, the time point of second procedure was taken. The Kaplan-Meier survival estimates method was used to calculate DFS as well as OS, and significant differences were examined using the log-rank-test. A difference was considered to be significant when the $p$ value was less than 0.05 . The analysis was performed using the statistical software Statistica (StatSoft, Tulsa, OK, USA). Subgroups of patients were specified as oncologically comparable when there was no statistical significant difference in all of the following parameters: primary tumor location (colon versus rectum), T-stage and N-stage of the primary tumor, UICCstage, percentage of patients receiving preoperative chemotherapy before LR, distribution of clinical risk scores (Fong and Nordlinger), and percentage of patients receiving postoperative anti-cancer therapy in adjuvant intent.

\section{Ethical considerations}

All human interventions were performed in accordance with the ethical standards laid down in the Declaration of Helsinki as revised in 1983 and have been approved by the regional ethical committee. All patients gave their written informed consent.

\section{Results}

Figure 1 summarizes the stratification of the study population into the different treatment arms. The mean age of our study population was $60.7 \pm 9.4$ years. The mean number of CRC-LM identified by initial staging procedures were $3 \pm 1.8$ (range 1-7; median 2) with a mean diameter of $3.3 \pm 2.2 \mathrm{~cm}$ (range 1-12; median $3 \mathrm{~cm}$ ).

Preoperative chemotherapy

Detailed data on the applied drug combinations are displayed in Table 2. The heterogeneity of regimens has to be attributed to the diversity of referring medical oncologists. The 43 patients received a mean number of $5.6 \pm 4.4$ (median 4; range 2-25) cycles of 5FU-based chemotherapy regimen which in $95 \%$ (41 patients) had been intensified by the addition of oxaliplatin or irinotecan and in $42 \%$ also by one of the monoclonal antibodies cetuximab or bevacizumab. In particular, $5 \mathrm{FU} /$ folinic acid + oxaliplatin (FOLFOX) had been chosen in $40 \%$ of patients 


\section{Patients with bilobar CRC-LM (n=64)}

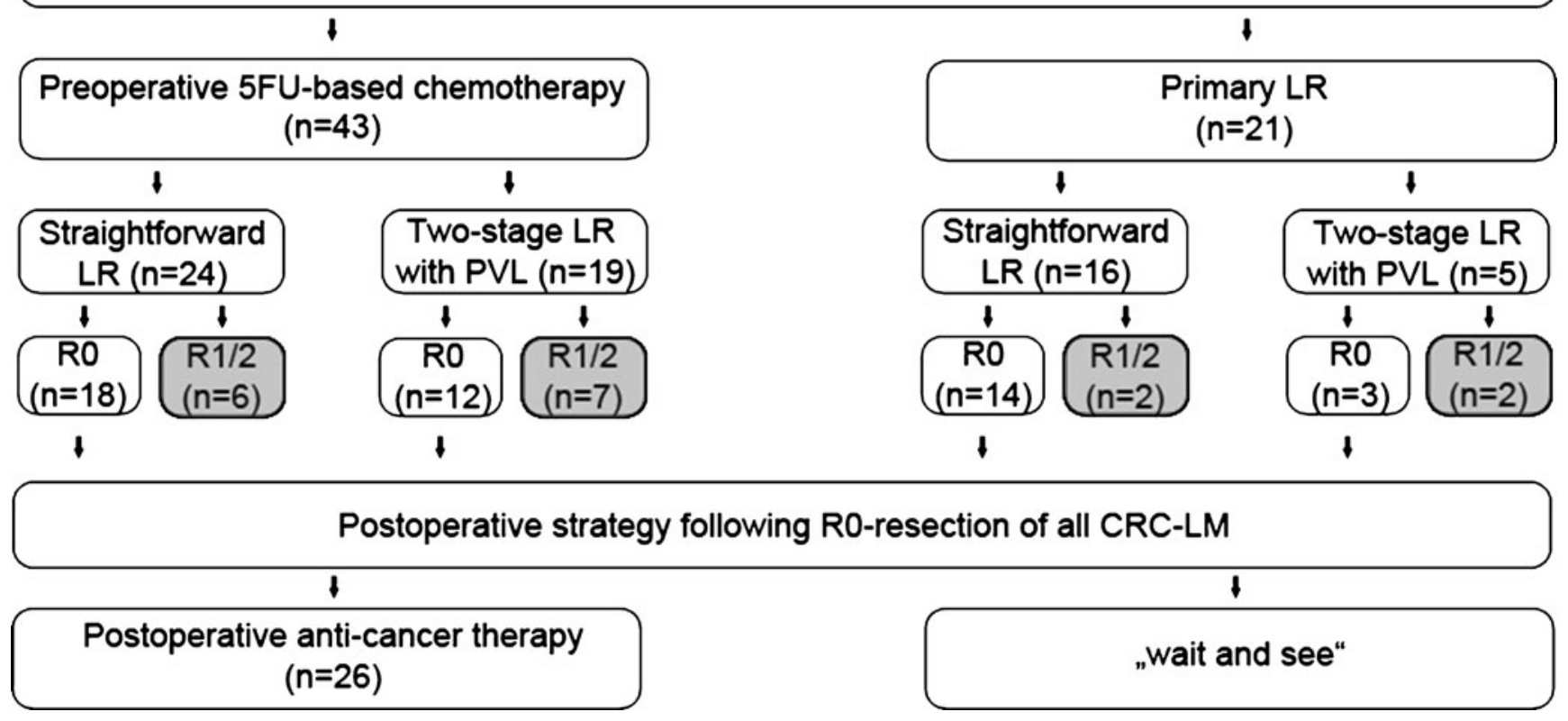

Fig. 1 Therapy algorithm of study population with bilobar CRC-LM $(n=64)$. Study population. Patients with incomplete (R1/R2) resection received palliative chemotherapy postoperatively. Abbreviations: $C R C-L M$ colorectal liver metastases, $L R$ liver resection, $P V L$ portal vein ligation

while 5FU/folinic acid + irinotecan (FOLFIRI) had been used only in $7 \%$. Based on RECIST criteria, preoperative chemotherapy resulted in partial remission in 16 and stable disease in 20 patients. Seven patients had minor progressive disease limited to the liver. However, all patients were deemed technically resectable at restaging prior to extensive surgery in our department.

Table 2 Preoperative chemotherapy regimens for downsizing of initially unresectable bilobar colorectal liver metastases $(n=43)$

\begin{tabular}{|c|c|c|}
\hline \multirow[t]{2}{*}{ Chemotherapy regimen } & \multicolumn{2}{|c|}{ Patients } \\
\hline & No. & $\%$ \\
\hline 5FU/folinic acid alone & 2 & 5 \\
\hline Additional oxaliplatin & 17 & 40 \\
\hline Additional oxaliplatin + cetuximab & 4 & 9 \\
\hline Additional oxaliplatin + bevacizumab & 1 & 2 \\
\hline Additional irinotecan & 3 & 7 \\
\hline Additional irinotecan + cetuximab & 3 & 7 \\
\hline Additional irinotecan + bevacizumab & 6 & 14 \\
\hline Additional oxaliplatin + sequential irinotecan ${ }^{\mathrm{a}}$ & 3 & 7 \\
\hline Additional oxaliplatin + sequential irinotecan ${ }^{\mathrm{a}}+$ cetuximab & 2 & 5 \\
\hline $\begin{array}{l}\text { Additional oxaliplatin }+ \text { sequential irinotecan }{ }^{\mathrm{a}}+ \\
\text { bevacizumab }\end{array}$ & 2 & 5 \\
\hline
\end{tabular}

5FU 5-fluorouracil

${ }^{\text {a }}$ Either oxaliplatin followed by irinotecan or vice versa

\section{Surgical procedures}

Operative procedures are summarized in Table 3. The decision on two-stage hepatectomy was made prior to the first procedure in all 24 patients affected. The corresponding perioperative complications are displayed in detail in Table 4. The surgical morbidity and mortality rates were $33 \%$ and $1.5 \%$, respectively.

In 51 patients, macroscopically complete resection of all detectable CRC-LM was achieved. Of these, six patients were treated only by unilobar LR $(1 \times$ minor LR of 1 segment, $5 \times$ right hemihepatectomy of segment $\mathrm{V}-\mathrm{VIII}$ ) based on downsizing of CRC-LM by preoperative chemotherapy. The histopathological examination of the surgical specimens revealed vital tumor cells at the resection margin (R1-resection) in four patients (8\%). R0-Resection rates in otherwise oncologically comparable patients treated by straightforward resection versus two-stage hepatectomy were not significantly different $(p=0.1511)$.

The surgical approach was non-curative because of unresectable disease in the remaining 13 patients (20\%). Eight of these 13 patients were treated within a two-stage concept using PVL, but resection of all CRC-LM could not be performed as they experienced unresectable tumor progression $(n=7)$ or denied second-stage procedure $(n=1)$. Additionally, four patients were surgically explored, but LR was also cancelled due to intraoperatively diagnosed unresectable disease. In one patient, multiple non-anatomical resections had to be combined with RFA as several metastases were unresectable. 
Table 3 Surgical procedures

\begin{tabular}{|c|c|c|}
\hline \multirow[t]{2}{*}{ Procedure } & \multicolumn{2}{|c|}{ Patients } \\
\hline & No. & $\%$ \\
\hline Surgical exploration only ${ }^{a}$ & 7 & 11 \\
\hline Minor resection ${ }^{\mathrm{b}}$ & 3 & \\
\hline Multiple minor resections ${ }^{\mathrm{c}}$ & 13 & 20 \\
\hline Bisegmentectomy segment II/III ${ }^{\mathrm{d}}$ & 3 & \\
\hline $\begin{array}{l}\text { Bisegmentectomy segment II/III + non-anatomical } \\
\text { resection right hemiliver }\end{array}$ & 3 & \\
\hline Central liver resection & 5 & \\
\hline $\begin{array}{l}\text { Left hemihepatectomy }+ \text { non-anatomical resection } \\
\text { right hemiliver }\end{array}$ & 1 & \\
\hline Right hemihepatectomy & 5 & \\
\hline Right hemihepatectomy + minor resection left hemiliver & 5 & \\
\hline Extended right hemihepatectomy ${ }^{\mathrm{e}}$ & 7 & 11 \\
\hline Right trisegmentectomy & 12 & 19 \\
\hline
\end{tabular}

Of the seven patients with surgical exploration only, four had intraoperatively diagnosed unresectable disease, and three were treated within a two-stage concept but experienced unresectable tumor progression during the interval between the PVL and the scheduled second exploration. Six patients were treated only by unilobar liver resection $(1 \times$ minor resection, $5 \times$ right hemihepatectomy) based on downsizing of CRC-LM by preoperative chemotherapy. The remaining unilobar resections were performed during first stage procedure within the twostage hepatectomy concept but patients did not proceed to second-stage procedure or were unresectable at the time of re-exploration

${ }^{\mathrm{a}}$ Together with portal vein ligation in three patients

${ }^{\mathrm{b}}<2$ segments

${ }^{\mathrm{c}}$ In one patient combined with radiofrequency ablation

${ }^{d}$ Together with portal vein ligation during initial operation within twostage hepatectomy

${ }^{\mathrm{e}}$ Extended by partial resection of segment IV

Postoperative therapy in adjuvant intent

Of the 47 patients that had histopathologically confirmed R0 resection of biCRC-LM, 21 patients did not receive postoperative anti-cancer therapy in adjuvant intent but had routine follow-up every 3 months according to national guidelines [15]. Twenty-six patients received either 3 cycles of 5FUbased systemic chemotherapy $(n=5 ; 2 \times 5 \mathrm{FU} /$ folinic acid, $2 \times 5 \mathrm{FU} /$ folinic acid + oxaliplatin, $1 \times 5 \mathrm{FU} /$ folinic acid + irinotecan) or anti-CEA-radioimmunotherapy with ${ }^{131}$ I-labetuzumab $(n=21 ; 10 \times$ single dose, $11 \times$ repeated dose $)$ in a dose of $40-50 \mathrm{mCi} / \mathrm{m}^{2}$.

Follow-up

Median DFS for R0-resected patients $(n=47)$ was 8 months. There was no statistically significant difference in DFS ( $p=$ $0.650)$ and OS $(p=0.435)$ between the oncologically
Table 4 Postoperative complications

\begin{tabular}{llc}
\hline & Patients & \\
\cline { 2 - 3 } & No. & \\
\hline Morbidity & & \\
Cardiopulmonary & 4 & 6 \\
Wound healing & 5 & 8 \\
Biliary leakage & 7 & 5 \\
Liver insufficiency $^{\mathrm{a}}$ & 3 & 3 \\
Reoperation $^{\mathrm{b}}$ & 2 & 1.5 \\
Mortality & 1 &
\end{tabular}

${ }^{\mathrm{a}}$ Defined by prothrombin time $<50 \%$ and serum bilirubin $>50 \mu \mathrm{mol} / 1$ on post-op day 5 (Balzan et al. [12])

${ }^{\mathrm{b}}$ Both reoperations were performed due to wound healing complications

Percentage is related to whole study population

comparable patients treated within two-stage concepts with PVL compared to those having straightforward LR.

Thirty out of 47 patients (64\%) developed metastatic recurrence after potentially curative R0-LR. Being afflicted in 21 of these 30 patients $(70 \%)$, the liver was the main site of metastatic recurrence. In particular, metastases were confined to the liver in 16 and involved simultaneous liver and lung in five patients, respectively. Potentially curative re-resection of all metastases could be achieved in nine of the 30 patients $(30 \%)$. In these nine patients, the metastatic recurrence was limited to either the liver or the lung.

The median OS of the 47 R0-resected patients was 38 months. This was significantly higher $(p=0.035)$ than of patients with incomplete (R1/R2) resection followed by palliative systemic 5FU-based chemotherapy (19 months, Fig. 2). Stratifying the patients following R0-LR in those with versus those without postoperative anti-cancer therapy in adjuvant intent resulted in two otherwise oncologically comparable subgroups. Receiving anti-cancer therapy postoperatively was not associated with a significant advantage in DFS $(p=0.269)$ but in OS $(p=0.048)$ compared to the "wait and see" strategy (Fig. 3) in those patients who denied postoperative anti-cancer treatment on behalf of the national guidelines.

\section{Discussion}

There is a consensus between medical oncologists and hepatobiliary surgeons that resectability of all CRC-LM is the key issue in curative intended treatment concepts [1, 16]. However, the individual assessment of resectability might differ even between experienced hepatobiliary surgeons. This has been demonstrated by the recently 


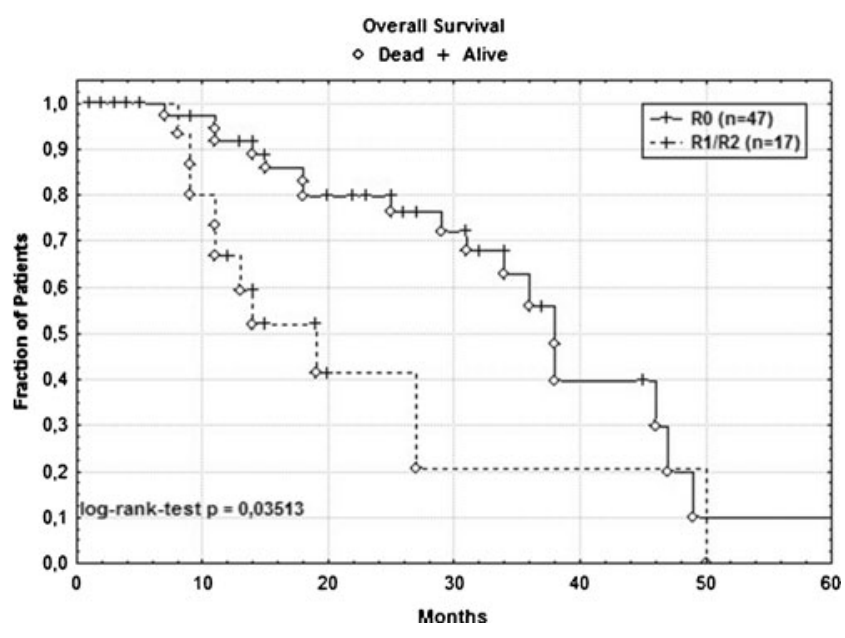

Fig. 2 Kaplan-Meier plot of cancer-specific overall survival in 47 patients with complete (R0) resection and 17 patients with $\mathrm{R} 1 / 2$ resection. With 38 versus 19 months, the median survival for R0-resected patients was significantly higher $(p=0.035)$

presented analysis of the blinded surgical review within the CELIM trial [6]. In that review, critical disagreement (one surgeon classifying a lesion as resectable and another as non-resectable) occurred in 131 out of 1,913 decision pairs $(6.8 \%)$. This possibility of disagreement gives explanation to the assessment of secondary resectability in those patients of our study that experienced tumor progression under preoperative chemotherapy.

However, to convert patients with initially unresectable biCRC-LM into those having a resectable disease requires the expertise of an interdisciplinary team and often the application of multiple therapeutical regimens resulting in individualized therapy algorithms.

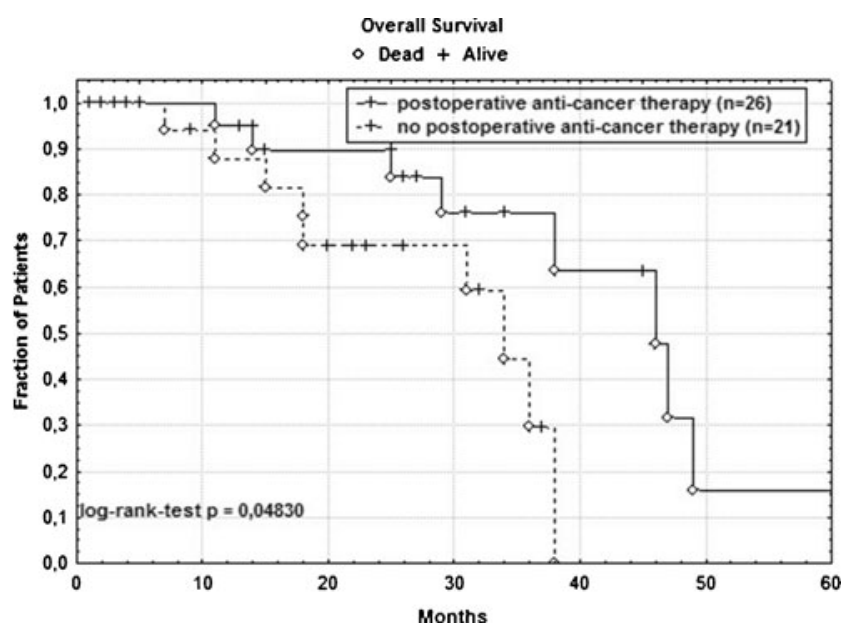

Fig. 3 Kaplan-Meier plot of cancer-specific overall survival in 47 R0-resected patients with bilobar CRC-LM. A significant survival benefit $(p=0.048)$ could be observed for those patients treated by postoperative anti-cancer therapy
Influence of two-stage hepatectomy with PVL on resection rate and survival

R0-resection rates of straightforward $\mathrm{LR} \pm$ preoperative 5FUbased chemotherapy were $75 \%$ and $88 \%$, respectively. In contrast, following two-stage hepatectomy with PVL \pm preoperative $5 \mathrm{FU}$-based chemotherapy, R0-resection rates of $63 \%$ and $60 \%$ were achieved. Although the latter are noticeably lower, the difference was not statistically significant $(p=0.1511)$. The observed resection rates of two-stage hepatectomy are comparable to those published by other authors [17] who also did not use tumor-directed therapy between first- and second-stage procedures. Recently, Wicherts et al. [18] published their long-term results of two-stage hepatectomy for initially unresectable CRC-LM $(n=59)$. Interestingly, the majority of patients experiencing R0-resection $(n=41)$ had systemic chemotherapy not only before (95\%) but also between (78\%) and after (78\%) the two surgical procedures. In contrast, none of the 18 patients failing two-stage hepatectomy had systemic chemotherapy after the first-stage procedure. Unfortunately, the authors did not provide detailed data on the administered chemotherapeutic regimens. A benefit of chemotherapy between the two surgical procedures has been also supported by Lygidakis et al. [19] who reported a $100 \%$ secondary R0-resection rate administering a 5FU-based regional intra-arterial chemotherapy intensified by oxaliplatin, irinotecan, and bevacizumab. Given that tumor progression occurs concomitantly to liver hypertrophy [7, 20], the data of Wicherts et al. [18], Lygidakis et al. [19] as well as our data argue for tumordirected therapy during liver hypertrophy which needs to be evaluated in randomized controlled phase II clinical trials.

Our data clearly indicate that DFS as well as OS in R0resected patients treated by two-stage hepatectomy do not significantly differ from those patients being with straightforward LR ( $p=0.650$ and 0.435 , respectively). These data confirm recently published results of Mueller et al. [21]. Although they did not focus on biCRC-LM, they reported 5 -year OS rates after R0-LR with PVO of $45.53 \%$ versus $37.67 \%$ without $\mathrm{PVO}(p=0.625)$. These comparable results are remarkable taking the PVO-related induction of tumor growth into consideration [7, 20]. Multiple factors could be associated with this finding: the considerable interindividual variation in the intensity of PVO-related tumor growth induction which in our experience does not correlate with the extend of volume gain on an individual basis [7], the use of preoperative systemic chemotherapy which has been considered to independently prolong DFS [22], and the administration of postoperative systemic therapy in adjuvant intent. Furthermore, PVO might function as a selection factor. Patients with highly aggressive diseases likely retain unresectable due to progression during liver hypertrophy and do not proceed to R0-LR. In 
contrast, if initially undetectable tumor cell clusters become detectable and accordingly secondarily resectable because of the PVO-related growth induction, this might result in a reduced number of residual tumor cell formations in the remnant liver. Besides, the comparable OS rates, however, can in part be attributed to our aggressive multimodal approach even for metastatic recurrence after R0-LR.

\section{Overall survival}

We achieved a median OS of 38 months following R0-LR in patients with biCRC-LM. This is significantly higher ( $p=0.035)$ than in those patients with $\mathrm{R} 1 / \mathrm{R} 2-\mathrm{LR}$ followed by-palliative systemic 5FU-based chemotherapy. The latter is comparable to first-line treatment, e.g., with 5FU, leucovorin, and irinotecan [23]. The early dissociation of the corresponding survival curves (Fig. 2) reflects the advanced disease stage of patients with biCRC-LM and the limited effectiveness of systemic chemotherapy. Hurwitz et al. have shown in 813 patients that adding bevacizumab to systemic 5FU-based first-line chemotherapy resulted in an extended median survival of 20.3 months in patients with metastatic colorectal cancer [24] However, Tol et al. [25] recently published their results comparing capecitabine, oxaliplatin, and bevacizumab versus the same regimen plus cetuximab in 775 patients with previously untreated metastatic colorectal cancer. They observed no significant benefit in median OS by adding the second antibody (20.3 versus 19.4 months, $p=0.16$ ), but reported mortality rates of $1.9 \%$ (without cetuximab) and $2.7 \%$ (with cetuximab) and incidences of any grade 3 or 4 adverse event of $73.2 \%$ (without cetuximab) and $81.7 \%$ (with cetuximab), respectively. Compared to these chemotherapeutic regimens, surgical exploration offers the chance of curation, and the corresponding morbidity as well as mortality rates are similar or even lower (33\% and $1.5 \%$, respectively, in our study). Therefore, attempting LR with discontinuation of systemic 5FU-based chemotherapy is not adversarial in patients with biCRC-LM if R0-LR seems to be achievable.

Survival benefit of postoperative anti-cancer therapy in adjuvant intent

The observed median DFS of 8 months and OS of 38 months following R0-LR in patients with biCRC-LM are encouraging and can be attributed in part to the administration of postoperative anti-cancer therapy in adjuvant intent. This is highlightened by Fig. 3 indicating that patients exposed to systemic therapy had a significantly longer OS $(p=0.048)$ compared to those with routine follow-up only. Interpreting the survival curves, the effect of systemic therapy in adjuvant intent became apparent
20 months after R0-LR and resulted in a survival benefit of approximately 12 months in the long-term follow-up. Although the number of patients in our study is small, this survival benefit is encouraging and underlines the importance of a multimodal treatment in these patients. Previous retrospective analyses have supported the benefit of such therapy. By also including patients with unilobar disease into their study population of 171 patients, Portier et al. [26] observed a significantly longer DFS for those 86 patients treated by postoperative 5FU-based chemotherapy. However, they failed to demonstrate a significant benefit on OS. In contrast, Parks et al. [27] demonstrated in their pooled analysis that patients subjected to postoperative systemic 5FU-based chemotherapy experienced an improved OS. Additionally, Nordlinger et al. [22] recently published their data on perioperative FOLFOX4 chemotherapy in patients with resectable CRC-LM and reported an improvement of DFS within the first 2 years following LR for the chemotherapy group. Despite these studies, no prospective randomized trial has evaluated the survival benefit of intensified postoperative 5FUbased chemotherapy. In our study, the postoperative regimen had been heterogeneous. However, the data argue for a survival benefit of systemic therapy in adjuvant intent. Facing the lack of evidence in this important question, we hardly advocate a randomized controlled trial comparing systemic 5FU-based chemotherapy with specific anti-tumor therapies, e.g., antiCEA-radioimmunotherapy as postoperative therapy after R0-resection of CRC-LM.

\section{Conclusion}

The significantly higher OS following R0-resection versus R1/R2-LR justifies extended surgery within multimodal treatment concepts in patients with biCRC-LM. The use of two-stage hepatectomy did negatively affect neither R0-resection rate nor DFS and OS after complete LR. The administration of postoperative anti-cancer therapy was associated with a significant benefit in OS. Future controlled clinical studies should focus on innovative targeted treatment options especially for those patients persisting unresectable nowadays and on type and benefit of postoperative anti-cancer therapy in adjuvant intent.

Acknowledgments We thank Prof. Dr. D.M. Goldenberg (Garden State Cancer Center, Center for Molecular Medicine and Immunology, Belleville NJ, USA) not only for his critical comments on our manuscript but also for his continuous efforts to support the collaboration of oncological surgeons and nuclear medicine specialists aiming to develop innovative treatment options for patients with metastatic colorectal cancer. 
Open Access This article is distributed under the terms of the Creative Commons Attribution Noncommercial License which permits any noncommercial use, distribution, and reproduction in any medium, provided the original author(s) and source are credited.

\section{References}

1. Abdalla EK, Adam R, Bilchik AJ, Jaeck D, Vauthey JN, Mahvi D (2006) Improving resectability of hepatic colorectal metastases: expert consensus statement. Ann Surg Oncol 13: $1271-1280$

2. Abdalla EK, Vauthey JN, Ellis LM, Ellis V, Pollock R, Broglio KR, Hess K, Curley SA (2004) Recurrence and outcomes following hepatic resection, radiofrequency ablation, and combined resection/ablation for colorectal liver metastases. Ann Surg 239:818-827

3. Gayowski TJ, Iwatsuki S, Madariaga JR, Selby R, Todo S, Irish W, Starzl TE (1994) Experience in hepatic resection for metastatic colorectal cancer: analysis of clinical and pathologic risk factors. Surgery 116:703-710

4. Shimada H, Tanaka K, Matsuo K, Togo S (2006) Treatment for multiple bilobar liver metastases of colorectal cancer. Langenbeck's Arch Surg 391:130-142

5. Adam R (2003) Chemotherapy and surgery: new perspectives on the treatment of unresectable liver metastases. Ann Oncol 14:ii13-ii16

6. Folprecht G, Gruenberger T, Hartmann JT, Lordick F, Stoehlmacher J, Bechstein W, Ockert D, Herrmann T, Liersch T, Koehne CH (2010) Cetuximab plus FOLFOX6 or cetuximab plus FOLFIRI as neoadjuvant treatment of nonresectable colorectal liver metastases: a randomized multicenter study (CELIM-study). Lancet Oncol $11: 38-47$

7. Homayounfar K, Liersch T, Schuetze G, Niessner M, Goralczyk AD, Meller J, Langer C, Ghadimi BM, Becker H, Lorf T (2009) Two-stage hepatectomy (R0) with portal vein ligation-towards curing patients with extended bilobar colorectal liver metastases. Int J Colorectal Dis 24:409-418

8. Jaeck D, Oussoultzoglou E, Rosso E, Greget M, Weber JC, Bachellier P (2004) A 2-stage hepatectomy procedure combined with portal vein embolization to achieve curative resection for initially unresectable multiple and bilobar colorectal liver metastases. Ann Surg 240:1037-1051

9. Nikfarjam M, Shereef S, Kimchi ET, Gusani NJ, Jiang Y, Avella DM, Mahraj RP, Staveley-O'Carroll KF (2009) Survival outcomes of patients with colorectal liver metastases following hepatic resection or ablation in the era of effective chemotherapy. Ann Surg Oncol $16: 1860-1867$

10. Liersch T, Meller J, Kulle B, Behr TM, Markus P, Langer C, Ghadimi BM, Wegener WA, Kovacs J, Horak ID, Becker H, Goldenberg DM (2005) Phase II trial of carcinoembryonic antigen radioimmunotherapy with 131I-labetuzumab after salvage resection of colorectal metastases in the liver: five-year safety and efficacy results. J Clin Oncol 23:6763-6770

11. Liersch T, Meller J, Bittrich M, Kulle B, Becker H, Goldenberg DM (2007) Update of carcinoembryonic antigen radioimmunotherapy with (131)I-labetuzumab after salvage resection of colorectal liver metastases: comparison of outcome to a contemporaneous control group. Ann Surg Oncol 14:2577-2590
12. Therasse P, Arbuck SG, Eisenhauer EA, Wanders J, Kaplan RS, Rubinstein L, Verweij J, Van Glabbeke M, van Oosterom AT, Christian MC, Gwyther SG (2000) New guidelines to evaluate the response to treatment in solid tumors. J Natl Cancer Inst 92:205216

13. Truant S, Oberlin O, Sergent G, Lebuffe G, Gambiez L, Ernst O, Pruvot FR (2007) Remnant liver volume to body weight ratio $>0.5 \%$ : a new cut-off to estimate postoperative risks after extended resection in noncirrhotic liver. J Am Coll Surg 204:22-33

14. Balzan S, Belghiti J, Farges O, Ogata S, Sauvanet A, Delefosse D, Durand F (2005) The "50-50 criteria" on postoperative day 5: an accurate predictor of liver failure and death after hepatectomy. Ann Surg 242:824-829

15. Schmiegel W, Pox C, Adler G, Fleig W, Folsch UR, Fruhmorgen P, Graeven U, Hohenberger W, Holstege A, Junginger T, Kühlbacher T, Porschen R, Propping P, Riemann JF, Sauer R, Sauerbruch T, Schmoll HJ, Zeitz M, Selbmann HK (2004) S3Guidelines conference "Colorectal Carcinoma" 2004. Z Gastroenterol 42:1129-1177

16. Folprecht G, Grothey A, Alberts S, Raab HR, Koehne CH (2005) Neoadjuvant treatment of unresectable colorectal liver metastases: correlation between tumour response and resection rates. Ann Oncol 16:1311-1319

17. Pamecha V, Glantzounis G, Davies N, Fusai G, Sharma D, Davidson B (2009) Long-term survival and disease recurrence following portal vein embolisation prior to major hepatectomy for colorectal metastases. Ann Surg Oncol 16:1202-1207

18. Wicherts DA, Miller R, de Haas RJ, Bitsakou G, Vibert E, Veilhan LA (2008) Long-term results of 2-stage hepatectomy for irresectable colorectal cancer liver metastases. Ann Surg 248: 994-1005

19. Lygidakis NJ, Bhagat AD, Vrachnos P, Grigorakos L (2007) Challenges in everyday surgical practice: synchronous bilobar hepatic colorectal metastases - newer multimodality approach. Hepatogastroenterology 54:1020-1024

20. Kokudo N, Tada K, Seki M, Ohta H, Azekura K, Ueno M, Ohta K, Yamaguchi T, Matsubara T, Takahashi T, Nakajima T, Muto T, Ikari T, Yanagisawa A, Kato Y (2001) Proliferative activity of intrahepatic colorectal metastases after preoperative hemihepatic portal vein embolization. Hepatology 34:267-272

21. Mueller L, Hillert C, Möller L, Krupski-Berdien G, Rogiers X, Broering DC (2008) Major hepatectomy for colorectal metastases: is preoperative portal occlusion an oncological risk factor? Ann Surg Oncol 15:1908-1917

22. Nordlinger B, Sorbye H, Glimelius B, Poston GJ, Schlag PM, Rougier P, Bechstein WO, Primrose JN, Walpole ET, Finch-Jones M, Jaeck D, Mirza D, Parks RW, Collette L, Praet M, Bethe U, Van Cutsem E, Scheithauer W, Gruenberger T (2008) Perioperative chemotherapy with FOLFOX4 and surgery versus surgery alone for resectable liver metastases from colorectal cancer (EORTC Intergroup trial 40983): a randomised controlled trial. Lancet 371:1007-1016

23. Saltz LB, Cox JV, Blanke C, Rosen LS, Fehrenbacher L, Moore MJ, Maroun JA, Ackland SP, Locker PK, Pirotta N, Elfring GL, Miller LL (2000) Irinotecan plus fluorouracil and leucovorin for metastatic colorectal cancer. Irinotecan Study Group. N Engl J Med 343:905-914

24. Hurwitz H, Fehrenbacher L, Novotny W, Cartwright T, Hainsworth J, Heim W, Berlin J, Baron A, Griffing S, Holmgren E, Ferrara N, Fyfe G, Rogers B, Ross R, Kabbinavar F (2004) Bevacizumab plus irinotecan, fluorouracil, and leucovorin for metastatic colorectal cancer. N Engl J Med 350:2335-2342 
25. Tol J, Koopman M, Cats A, Rodenburg CJ, Creemers GJM, Erdkamp SJG, FL VAH, van Groeningen CJ, Sinnige HA, Richel DJ, Voest EE, Dijkstra JR, Vink-Börger ME, Antonini NF, Mol L, van Krieken JH, Dalesio O, Punt CJ (2009) Chemotherapy, bevacizumab, and cetuximab in metastatic colorectal cancer. $\mathrm{N}$ Engl J Med 360:563-572

26. Portier G, Elias D, Bouche O, Rougier P, Bosset JF, Saric J, Belghiti J, Piedbois P, Guimbaud R, Nordlinger B, Bugat R, Lazorthes F, Bedenne L (2006) Multicenter randomized trial of adjuvant fluorouracil and folinic acid compared with surgery alone after resection of colorectal liver metastases: FFCD ACHBTH AURC 9002 trial. J Clin Oncol 24:49764982

27. Parks R, Gonen M, Kemeny N, Jarnagin W, D'Angelica M, DeMatteo R, Garden OJ, Blumgart LH, Fong Y (2007) Adjuvant chemotherapy improves survival after resection of hepatic colorectal metastases: analysis of data from two continents. J Am Coll Surg 204:753-761 